(1)

CrossMark

\title{
Integrating high dose inhaled corticosteroids into oral corticosteroids stewardship
}

\author{
Arnaud Bourdin ${ }^{1,2}$, Carey Suehs $\mathbb{1}^{1,3}$ and Jérémy Charriot (10 ${ }^{1}$ \\ Affiliations: ${ }^{1}$ Dept of Respiratory Diseases, Univ Montpellier, CHU Montpellier, Montpellier, France. ${ }^{2}$ Univ \\ Montpellier, PhyMedExp, INSERM, CNRS CHU Montpellier, Montpellier, France. ${ }^{3}$ Dept of Medical Information, \\ Univ Montpellier, CHU Montpellier, Montpellier, France.
}

Correspondence: Arnaud Bourdin, Dept of Respiratory Diseases, Montpellier University Hospital, Hôpital Arnaud de Villeneuve, 371, av. du Doyen Gaston Giraud, 34295 Montpellier Cedex 5, France. E-mail: a-bourdinachu-montpellier.fr

@ERSpublications

If high doses of ICS are equivalent to low dose OCS, they should be considered as such http://bit.ly/2CYssfB

Cite this article as: Bourdin A, Suehs C, Charriot J. Integrating high dose inhaled corticosteroids into oral corticosteroids stewardship. Eur Respir J 2020; 55: 1902193 [https://doi.org/10.1183/13993003.02193-2019].

Uncertainties still surround high dose inhaled corticosteroids (ICS) use in asthma. In hindsight, certain aspects of the ICS development story can help elucidate why. In 1973, CAMERON et al. [1] signed a brilliant paper reporting the results of a double blind, randomised controlled trial demonstrating the oral corticosteroid (OCS)-sparing effect of ICS as the primary outcome. A few years later, the assessment of this benefit was mitigated when a complete weaning of OCS remained unachievable [2]. The benefit of ICS was therefore understood to be mostly based on an improved safety profile purportedly due to reduced systemic diffusion. Thus, the understanding of how ICS was of any benefit to asthma patients when compared to OCS was mostly based on a greater safety profile supposedly due to a reduced systemic diffusion. Similarly, topically administered corticosteroids were also developed in the same time period for diseases affecting the skin, the eyes, the nose or the joints. As for ICS, whether or not these formulations reduce corticosteroid-associated adverse events remains largely debated [3].

Outside rare conditions such as eosinophilic granulomatosis with polyangiitis, the systemic components of asthma are not obvious and the reasons driving requirements for systemic corticosteroids quite unknown [4]. Interestingly, multiple studies have raised safety concerns related to the use of ICS, especially at high doses [5]. Specific adverse-effect studies have focussed mainly on skin bruising or osteoporosis [6, 7]. However, hypothalamo-pituitary axis (HPA) suppression has been described for high-dose ICS since at least the 1990s [8, 9]. In a slightly provocative way, MAIJERs et al. [10] suggest in the present issue of the European Respiratory Journal that around two thirds of high-dose ICS effects are due to their systemic diffusion. To reach this point, they compiled studies where OCS-sparing effects attributed to ICS were studied, and then computed the meta-regression between the change in prednisone dose and the dose of ICS. Based on HPA suppression data, their findings suggest that at least $60 \%$ of the OCS dose reduction can be attributed to systemic absorption.

Although the conclusion put forward by MaIjers et al. [10] is limited by a paucity of studies and patients, it provides evidence that the systemic diffusion of ICS is far from minimised. Moreover, even though HPA suppression is not necessarily related to efficacy, the result is more consistent when considering the 
relatively homogeneous results observed with either budesonide or fluticasone. Thanks to these data, the distinction between Global Initiative for Asthma step 4 and step 3 is better justified, and a debate is justified on whether high doses of ICS should be placed just next to low OCS doses within step 5. Why "very high" doses of ICS are not providing clear advantages beyond "high" doses is a relevant mechanistic question [11]. Corticosteroid resistance is a paradox within the T2 pathway eventually overwhelmed by IL-4, -5 and -13 targeting drugs, even though their mechanisms of action largely overlap [12].

Beyond these observations and questions, the main practical conclusion from the paper by MAIJERS et al. [10] might be that high doses of ICS should be considered as potentially as harmful as low doses of OCS. In this context, the development of equivalencies between ICS and OCS dosing regimens will be necessary for describing the systemic distribution of ICS. It follows that methods for quantifying cumulative doses of corticosteroid treatment that appropriately take into account different modes of administration will take on increased importance as markers of disease severity and/or the success of corticosteroid-sparing strategies. A recent worldwide initiative aimed at reaching consensus around OCS tapering has recently finished data collection and is currently undergoing analysis [13]. Consensus statements related to high doses of ICS and cumulating doses of corticosteroids in general are particularly poised for discussion, and will likely affect the view of corticosteroid stewardship the asthma world stakeholders are currently promoting. Finally, like a little brother to OCS-sparing, ICS-sparing is attaining position as a relevant outcome in future trials, especially among asthma patients who are biological super-responders.

Conflict of interest: A. Bourdin reports receiving personal fees from Actelion, AstraZeneca, Boehringer Ingelheim, Chiesi Pharmaceuticals, GlaxoSmithKline, Novartis, Regeneron, Roche and Teva; receiving grants from Boehringer Ingelheim and GlaxoSmithKline; receiving non-financial support from Actelion, AstraZeneca, Boehringer Ingelheim, Novartis, Chiesi Pharmaceuticals, and Roche; and being an advisory board member for Actelion, AstraZeneca, Boehringer Ingelheim, Chiesi Pharmaceuticals, Gilead, GlaxoSmithKline, Novartis, Regeneron and Teva. C. Suehs has nothing to disclose. J. Charriot has nothing to disclose.

\section{References}

1 Cameron SJ, Cooper EJ, Crompton GK, et al. Substitution of beclomethasone aerosol for oral prednisolone in the treatment of chronic asthma. $\mathrm{Br}$ Med J 1973; 4: 205-207.

Herxheimer H. Should corticosteroid aerosols be used in severe chronic asthma? Thorax 1981; 36: 401-403

Adcock IM, Mumby S. Glucocorticoids. Handb Exp Pharmacol 2017; 237: 171-196.

Trevor JL, Deshane JS. Refractory asthma: mechanisms, targets, and therapy. Allergy 2014; 69: 817-827.

Heffler E, Madeira LNG, Ferrando M, et al. Inhaled corticosteroids safety and adverse effects in patients with asthma. J Allergy Clin Immunol Pract 2018; 6: 776-781.

6 Malo JL, Cartier A, Ghezzo H, et al. Skin bruising, adrenal function and markers of bone metabolism in asthmatics using inhaled beclomethasone and fluticasone. Eur Respir J 1999; 13: 993-998.

7 Roy A, Leblanc C, Paquette L, et al. Skin bruising in asthmatic subjects treated with high doses of inhaled steroids: frequency and association with adrenal function. Eur Respir J 1996; 9: 226-231.

8 Brown PH, Blundell G, Greening AP, et al. Hypothalamo-pituitary-adrenal axis suppression in asthmatics inhaling high dose corticosteroids. Respir Med 1991; 85: 501-510.

9 Broide J, Soferman R, Kivity S, et al. Low-dose adrenocorticotropin test reveals impaired adrenal function in patients taking inhaled corticosteroids. J Clin Endocrinol Metab 1995; 80: 1243-1246.

10 Maijers I, Kearns N, Harper J, et al. Oral steroid-sparing effect of high-dose inhaled corticosteroids in asthma. Eur Respir J 2020; 55: 1901147.

11 Adams NP, Jones PW. The dose-response characteristics of inhaled corticosteroids when used to treat asthma: an overview of Cochrane systematic reviews. Respir Med 2006; 100: 1297-1306.

$12 \mathrm{Wu}$ W, Bang S, Bleecker ER, et al. Multiview cluster analysis identifies variable corticosteroid response phenotypes in severe asthma. Am J Respir Crit Care Med 2019; 199: 1358-1367.

13 Suehs CM, Price D, Menzies-Gow A, et al. Expert Statements Concerning the Tapering of Oral Corticosteroids (OCS) for the Treatment of Asthma: A Delphi Consensus Study [OSF Registry]. https://osf.io/rikxd/ Date last updated: 4 April 2019. Date last accessed: 12 November 2019. 